\title{
Review Article \\ Review on the Pseudocomplex General Relativity and Dark Energy
}

\author{
Peter O. Hess $\mathbb{D}^{1,2}$ \\ ${ }^{1}$ Instituto de Ciencias Nucleares, UNAM, Circuito Exterior, C.U., A.P. 70-543, 04510, Mexico City, Mexico \\ ${ }^{2}$ Frankfurt Institute for Advanced Studies, Wolfgang Goethe University, Ruth-Moufang-Strasse 1, 60438 Frankfurt am Main, Germany
}

Correspondence should be addressed to Peter O. Hess; hess@nucleares.unam.mx

Received 26 March 2019; Accepted 30 May 2019; Published 20 June 2019

Guest Editor: Cesar A. Vasconcellos

Copyright (C) 2019 Peter O. Hess. This is an open access article distributed under the Creative Commons Attribution License, which permits unrestricted use, distribution, and reproduction in any medium, provided the original work is properly cited. The publication of this article was funded by SCOAP ${ }^{3}$.

\begin{abstract}
A review will be presented on the algebraic extension of the standard Theory of Relativity (GR) to the pseudocomplex formulation (pc-GR). The pc-GR predicts the existence of a dark energy outside and inside the mass distribution, corresponding to a modification of the GR-metric. The structure of the emission profile of an accretion disc changes also inside a star. Discussed are the consequences of the dark energy for cosmological models, permitting different outcomes on the evolution of the universe.
\end{abstract}

\section{Introduction}

The Theory of General Relativity (GR) [1] is one of the best tested known theories [2], mostly in solar system experiments. Also the loss of orbital energy in a binary system [3] was the first indirect proof for gravitational waves, which were finally detected in [4]. On April 10, The Event Horizon Telescope collaboration announced the first picture taken from the black hole in M87. This gives us an opportunity to compare results from the pc-GR to GR.

Nevertheless, the limits of GR may be reached when strong gravitational fields are present, which can lead to different interpretations of the sources of gravitational waves $[5,6]$.

A first proposal to extend GR was attempted by A. Einstein $[7,8]$ who introduced a complex valued metric $G_{\mu \nu}=$ $g_{\mu \nu}+i F_{\mu \nu}$, with $G_{\mu \nu}^{*}=G_{\nu \mu}$. The real part corresponds to the standard metric, while the imaginary part defines the electromagnetic tensor. With this, A. Einstein intended to unify GR with Electrodynamics. Another motivation to extend GR is published in $[9,10]$, where M. Born investigated on how to recover the symmetry between coordinates and momenta, which are symmetric in Quantum Mechanics but not in GR. To achieve his goal, he introduced also a complex metric, where the imaginary part is momentum dependent.
In [11] this was more elaborated, leading to the square of the length element $(c=1)$

$$
d \omega^{2}=g_{\mu \nu}\left[d x^{\mu} d x^{\nu}+l^{2} d u^{\mu} d u^{\nu}\right],
$$

which implies maximal acceleration (see also [12]). The interesting feature is that a minimal length "l" is introduced as a parameter and Lorentz symmetry is, thus, automatically maintained; no deformation to small lengths is necessary!

In [13] the GR was algebraically extended to a series of variables and the solutions for the limit of weak gravitational fields were investigated. As a conclusion, only real and pseudocomplex coordinates (called in [13] hyper-complex) make sense, because all others show either tachyon or ghost solutions, or both. Thus, even the complex solutions do not make sense. This was the reason to concentrate on the pseudocomplex extension.

In pc-GR, all the extended theories, mentioned in the last paragraph, are contained and the Einstein equations require an energy-momentum tensor, related to vacuum fluctuations (dark energy), described by an asymmetric ideal fluid [12]. Due to the lack of a microscopic theory, this dark energy is treated phenomenologically. One possibility is to choose it such that no event horizon appears or barely still exists. The reason to do so is that, in our philosophical understanding, no theory should have a singularity, even a coordinate singularity 
of the type of an event horizon encountered in a black hole. Though it is only a coordinate singularity, the existence of an event horizon implies that even a black hole in a nearby corner cannot be accessed by an outside observer. Its event horizon is a consequence of a strong gravitational field. Because no quantized theory of gravitation exists yet, we are led to the construction of models for the distribution of the dark energy.

In [14] the pc-GR was compared to the observation of the amplitude for the inspiral process. As found, the fall-off in $r$ of the dark energy has to be stronger than suggested in earlier publications. We will discuss this and what will change, in the main body of the text. We also will compare EHT observations with pc-GR, taking into account the low resolution of $20 \mu a s$, as obtained by the EHT. The main question is if one can discriminate between GR and pc-GR.

A general principle emerges; namely, that mass not only curves the space (which leads to the standard GR) but also changes the space- (vacuum-) structure in its vicinity, which in turn leads to an important deviation from the classical solution.

The consequences will be discussed in Section 3. There, also the cosmological effects are discussed, with different outcomes for the evolution of the dark energy as function of time/radius of the universe. Another application treats the interior of a stars, where first attempts will be reported on how to stabilize a large mass. In Section 4 conclusions will be drawn.

\section{Pseudocomplex General Relativity (pc-GR)}

An algebraic extension of GR consists in a mapping of the real coordinates to a different type, as, for example, complex or pseudocomplex $(\mathrm{pc})$ variables

$$
X^{\mu}=x^{\mu}+I y^{\mu}
$$

with $I^{2}= \pm 1$ and where $x^{\mu}$ is the standard coordinate in space-time and $y^{\mu}$ is the complex component. When $I^{2}=-1$ it denotes complex variables, while when $I^{2}=+1$ it denotes pseudocomplex (pc) variables. This algebraic mapping is just one possibility to explore extensions of GR.

In [13] all possible extensions of real coordinates in GR were considered. It was found that only the extension to pseudocomplex coordinates (called in [13] hyper-complex) makes sense, because all others lead to tachyon and/or ghost solutions, in the limit of weak gravitational fields.

In what follows, some properties of pseudocomplex variables are resumed, which is important to understand some of the consequences.

(i) The variables can be expressed alternatively as

$$
\begin{aligned}
& X^{\mu}=X_{+}^{\mu} \sigma_{+}+X_{-}^{\mu} \sigma_{-} \\
& \sigma_{ \pm}=\frac{1}{2}(1 \pm I) .
\end{aligned}
$$

(ii) The $\sigma_{ \pm}$satisfy the relations

$$
\begin{aligned}
\sigma_{ \pm}^{2} & =\sigma_{ \pm}, \\
\sigma_{+} \sigma_{-} & =0 .
\end{aligned}
$$

(iii) Due to the last property in (4), when multiplying one variable proportional to $\sigma_{+}$by another one proportional to $\sigma_{-}$, the result is zero; i.e., there is a zerodivisor. The variables, therefore, do not form a field but a ring.

(iv) In both zero-divisor components $\left(\sigma_{ \pm}\right)$the analysis is very similar to the standard complex analysis.

In pc-GR the metric is also pseudocomplex

$$
g_{\mu \nu}=g_{\mu \nu}^{+} \sigma_{+}+g_{\mu \nu}^{-} \sigma_{-} .
$$

Because $\sigma_{+} \sigma_{-}=0$ in each zero-divisor component one can construct independently a GR theory.

For a consistent theory, both zero-divisor components have to be connected! One possibility is to define a modified variational principle, as done in [15]. Alternatively, one can implement a constraint; namely, a particle should always move along a real path; i.e., the pseudocomplex length element should be real.

The infinitesimal pc length element squared is given by (see also [16])

$$
d \omega^{2}=g_{\mu \nu} d X^{\mu} d X^{\nu}=g_{\mu \nu}^{+} d X_{+}^{\mu} d X_{+}^{\nu} \sigma_{+}+g_{\mu \nu}^{-} d X_{-}^{\mu} d X_{-}^{\nu} \sigma_{-}
$$

as written in the zero-divisor components. In terms of the pseudoreal and pseudoimaginary components, we have

$$
\begin{aligned}
& d \omega^{2}=g_{\mu \nu}^{s}\left(d x^{\mu} d x^{\nu}+d y^{\mu} d y^{\nu}\right)+g_{\mu \nu}^{a}\left(d x^{\mu} d y^{\nu}\right. \\
& \left.+d y^{\mu} d x^{\nu}\right)+I\left[g_{\mu \nu}^{a}\left(d x^{\mu} d x^{\nu}+d y^{\mu} d y^{\nu}\right)\right. \\
& \left.+g_{\mu \nu}^{s}\left(d x^{\mu} d y^{\nu}+d y^{\mu} d x^{\nu}\right)\right],
\end{aligned}
$$

with $g_{\mu \nu}^{s}=(1 / 2)\left(g_{\mu \mu}^{+}+g_{\mu \nu}^{-}\right)$and $g_{\mu \nu}^{a}=(1 / 2)\left(g_{\mu \mu}^{+}-g_{\mu \nu}^{-}\right)$. The upper indices $s$ and $a$ refer to a symmetric and antisymmetric combination of the metrics. The case when $g_{\mu \nu}=g_{\mu \nu}^{+}=g_{\mu \nu}^{-}$, i.e., $g_{\mu \nu}^{a}=0$, leads to

$$
g_{\mu \nu}\left(d x^{\mu} d x^{\nu}+d y^{\mu} d y^{\nu}\right)+\operatorname{Ig}_{\mu \nu}\left(d x^{\mu} d y^{\nu}+d y^{\mu} d x^{\nu}\right) .
$$

Identifying $y^{\mu}=l u^{\mu}$, where $l$ is an infinitesimal length and $u^{\mu}$ is the 4 -velocity, one obtains the length element defined in [11]. It also contains the line element as proposed in $[9,10]$, where the $y^{\mu}$ is proportional to the momentum component $p^{\mu}$ of a particle. However, this identification of $y^{\mu}$ is only valid in a flat space, where the second term in (8) is just the scalar product of the 4-velocity $\left(u^{\mu}=d x^{\mu} / d \tau\right)$ to the 4 -acceleration $\left(y^{\mu}=d^{2} x^{\mu} / d \tau^{2}\right)$.

The connection between the two zero-divisor components is achieved, requiring that the infinitesimal length element squared in (7) is real; i.e., in terms of the $\sigma_{ \pm}$ components it is

$$
\left(\sigma_{+}-\sigma_{-}\right)\left(g_{\mu \nu}^{+} d X_{+}^{\mu} d X_{+}^{\nu}-g_{\mu \nu}^{-} d X_{-}^{\mu} d X_{-}^{\nu}\right)=0 .
$$


Using the standard variational principle with a Lagrange multiplier, to account for the constraint, leads to an additional contribution in the Einstein equations, interpreted as an energy-momentum tensor.

The action of the pc-GR is given by [16]

$$
S=\int d x^{4} \sqrt{-g}(\mathscr{R}+2 \alpha)
$$

where $\mathscr{R}$ is the Riemann scalar. The last term in the action integral allows to introduce the cosmological constant in cosmological models, where $\alpha$ has to be constant in order not to violate the Lorentz symmetry. This changes when a system with a uniquely defined center is considered, which has spherical (Schwarzschild) or axial (Kerr) symmetry. In these cases, the $\alpha$ is allowed to be a function in $r$, for the Schwarzschild solution, and a function in $r$ and $\vartheta$, for the Kerr solution.

The variation of the action with respect to the metric $g_{\mu \nu}^{ \pm}$ leads to the equations of motion

$$
\begin{aligned}
& \mathscr{R}_{\mu \nu}^{ \pm}-\frac{1}{2} g_{\mu \nu}^{ \pm} \mathscr{R}_{ \pm}=8 \pi T_{ \pm \mu \nu}^{\Lambda} \\
& \text { with } T_{ \pm \mu \nu}^{\Lambda}=\lambda u_{\mu} u_{\nu}+\lambda\left(\dot{y}_{\mu} \dot{y}_{\nu} \pm u_{\mu} \dot{y}_{\nu} \pm u_{\nu} \dot{y}_{\mu}\right)+\alpha g_{\mu \nu}^{ \pm},
\end{aligned}
$$

in the zero-divisor component, denoted by the independent unit-elements $\sigma_{ \pm}$. These equations still contain the effects of a minimal length parameter $l$, as shown in [11]. Because the effects of a minimal length scale are difficult to measure, maybe not possible at all, we neglect them, which corresponds to mapping the above equations to their real part, giving

$$
\mathscr{R}_{\mu \nu}-\frac{1}{2} g_{\mu \nu}^{ \pm} \mathscr{R}=8 \pi T_{\mu \nu}^{\Lambda}
$$

The $T_{\mu \nu}^{\Lambda}, \mathscr{R}_{\mu \nu}^{ \pm}$is real and is now given by [16]

$$
T_{\mu \nu, R}^{\Lambda}=\left(\rho_{\Lambda}+p_{\vartheta}^{\Lambda}\right) u_{\mu} u_{\nu}+p_{\vartheta}^{\Lambda} g_{\mu \nu}+\left(p_{r}^{\Lambda}-p_{\vartheta}^{\Lambda}\right) k_{\mu} k_{\nu},
$$

where $p_{\vartheta}^{\Lambda}$ and radial $p_{r}^{\Lambda}$ are the tangential and pressure, respectively. For an isotropic fluid we have $p_{9}^{\Lambda}=p_{r}^{\Lambda}=p^{\Lambda}$. The $u^{\mu}$ are the components of the 4-velocity of the elements of the fluid and $k^{\mu}$ is a space-like vector $\left(k_{\mu} k^{\mu}=1\right)$ in the radial direction. It satisfies the relation $u_{\mu} k^{\mu}=0$. The fluid is anisotropic due to the presence of $y_{\mu}$. The $\lambda$ and $\alpha$ are related to the pressures as [16]

$$
\begin{aligned}
\lambda & =8 \pi \tilde{\lambda}, \\
\alpha & =8 \pi \tilde{\alpha} \\
\tilde{\lambda} & =\left(p_{\vartheta}^{\Lambda}+\rho_{\Lambda}\right), \\
\tilde{\alpha} & =p_{\vartheta}^{\Lambda}, \\
\widetilde{\lambda} y_{\mu} y_{\nu} & =\left(p_{r}^{\Lambda}-p_{\vartheta}^{\Lambda}\right) k_{\mu} k_{\nu} .
\end{aligned}
$$

The reason why the dark energy outside a mass distribution has to be an anisotropic fluid is understood contemplating the Tolman-Oppenheimer-Volkov (TOV) equations [17] for an isotropic fluid: The TOV equations relate the derivative of the dark-energy pressure with respect to $r$ (for an isotropic fluid, the tangential pressure has to be the same as the radial pressure, i.e., $p_{\vartheta}^{\Lambda}=p_{r}^{\Lambda}=p^{\Lambda}$ ) to the dark energy density $\rho_{\Lambda}$. Assuming the isotropic fluid and that the equation of state for the dark energy is $p^{\Lambda}=-\rho_{\Lambda}$, the factor $\left(p^{\Lambda}+\rho_{\Lambda}\right)$ in the TOV equation for $d p^{\Lambda} / d r$ is zero; i.e., the pressure derivative is zero. As a result the pressure is constant and with the equation of state also the density is constant, which leads to a contradiction. Thus, the fluid has to be anisotropic, due to an additional term, allowing the pressure to fall off as a function on increasing distance. The additional term in the radial pressure $d p_{r}^{\Lambda} / d r$, added to the TOV equation, is given by $(2 / r) \Delta p^{\Lambda}=(2 / r)\left(p_{\vartheta}^{\Lambda}-p_{r}^{\Lambda}\right)[18]$.

For the density one has to apply a phenomenological model, due to the lack of a quantized theory of gravity. What helps is to recall one-loop calculations in gravity [19], where vacuum fluctuations result due to the non-zero back ground curvature (Casimir effect). Results are presented in [20], where at large distances the density falls off approximately as $1 / r^{6}$. The semiclassical Quantum Mechanics [19] was applied, which assumes a fixed back-ground metric and is thus only valid for weak gravitational fields (weak compared to the solar system). Near the Schwarzschild radius the field is very strong which is exhibited by a singularity in the energy density, which is proportional to $1 /(1-2 m / r)^{2}$, with $m$ a constant mass parameter [20].

Because we treat the vacuum fluctuations as a classical ideal anisotropic fluid, we are free to propose a different fall-off of the negative energy density, which is finite at the Schwarzschild radius. In earlier publications the density did fall-off proportional to $1 / r^{5}$. However, in [14] it is shown that this fall-off has to be stronger. Thus, in this contribution we will also discuss a variety of fall-offs as a function of a parameter $n$, i.e., proportional to $B_{n} / r^{n+2}$, where $B_{n}$ describes the coupling of the dark energy to the mass.

With the assumed density, the metric for the Kerr solution changes to $[21,22]$

$$
\begin{aligned}
& g_{00} \\
& =-\frac{r^{2}-2 m r+a^{2} \cos ^{2} \vartheta+B_{n} /(n-1)(n-2) r^{n-2}}{r^{2}+a^{2} \cos ^{2} \vartheta}, \\
& g_{11}=\frac{r^{2}+a^{2} \cos ^{2} \vartheta}{r^{2}-2 m r+a^{2}+B_{n} /(n-1)(n-2) r^{n-2}}, \\
& g_{22}=r^{2}+a^{2} \cos ^{2} \vartheta \\
& g_{33} \\
& =\left(r^{2}+a^{2}\right) \sin ^{2} \vartheta \\
& \quad+\frac{a^{2} \sin ^{4} \vartheta\left(2 m r-B_{n} /(n-1)(n-2) r^{n-2}\right)}{r^{2}+a^{2} \cos ^{2} \vartheta}
\end{aligned}
$$

$$
=\frac{-a \sin ^{2} \vartheta 2 m r+a\left(B_{n} /(n-1)(n-2) r^{n-2}\right) \sin ^{2} \vartheta}{r^{2}+a^{2} \cos ^{2} \vartheta},
$$




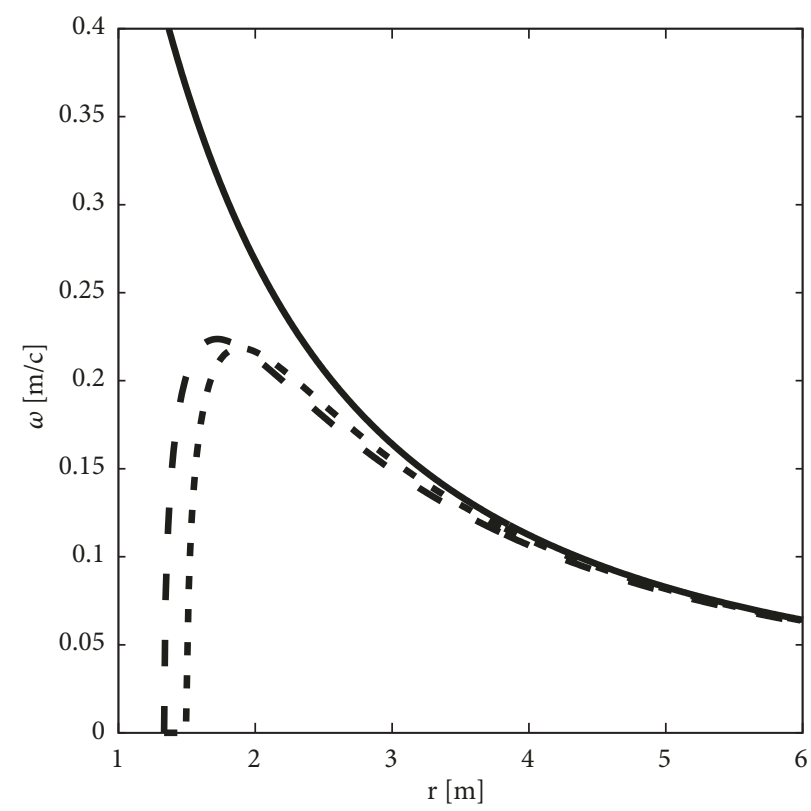

FIGURE 1: The orbital frequency of a particle in a circular orbit for the case GR (upper curve) and for $n=3$ (long dashed curve) and $n=4$ (short dashed curve) [12, 25].

where $0 \leq a \leq m$ is the spin parameter of the Kerr solution and $n=3,4, \ldots$ For $n=2$ the old ansatz is achieved. The Schwarzschild solution is obtained, setting $a=0$. The parameter $B_{n}=b_{n} m^{n}$ measures the coupling of the dark energy to the central mass. The definition of $n$ here is related to the $n_{N}$ in [14] by $n_{N}=n-1$.

When no event horizon is demanded, the parameter $B_{n}$ has a lower limit given by

$$
B_{n}>\frac{2(n-1)(n-2)}{n}\left[\frac{2(n-1)}{n}\right]^{n-1} \mathrm{~m}^{n}=b_{\max } \mathrm{m}^{n} \text {. }
$$

For the equal sign, an event horizon is located at

$$
r_{h}=\frac{2(n-1)}{n} \mathrm{~m}
$$

e.g., $4 / 3$ for $n=3$ and $3 / 2$ for $n=4$.

\section{Applications}

3.1. Motion of a Particle in a Circular Orbit. In [23] the motion of a particle in a circular orbit was investigated. This section was first discussed in $[24,25]$.

The main results are resumed in Figures 1 and 2. In Figure 1 the orbital frequency, in units of $c / m$, is depicted versus the radial distance $r$, in units of $m$, for a rotational parameter of $0.9 \mathrm{~m}$. The function for the orbital frequency, in prograde orbits, is given by

$$
\begin{aligned}
\omega_{n} & =\frac{1}{a+\sqrt{2 r / h_{n}(r)}} \\
h_{n}(r) & =\frac{2}{r^{2}}-\frac{n B_{n}}{(n-1)(n-2) r^{n+1}} .
\end{aligned}
$$

The upper curve in Figure 1 corresponds to GR while the two lower ones correspond to pc-GR with $n=3$ (dashed curve) and $n=4$ (dotted curve). The curve shows a maximum at

$$
r_{\omega_{\max }}=\left[\frac{n(n+2) b_{\max }}{6(n-1)(n-2)}\right]^{1 /(n-1)} \mathrm{m},
$$

which, for $b_{n}=b_{\max }$ as given in (16), is independent of the value of $a$, after which it falls off toward the center and reaches zero at $r_{h}$ (Eq. (17)), which is independent on the rotational parameter $a$. After the maximum the curve falls off toward smaller $r$. These features will be important for the understanding of the emission structure of an accretion disc (see next subsection).

As one can see, the difference between $n=3$ and $n=4$ is minimal and, thus, will not change the qualitative results as obtained for $n=3$ in former publications. The position of the maximum, which gives the position of the dark ring discussed below, is approximately the same in both cases. For $b_{n} \longrightarrow 0$ the curve approaches the one for GR.

In Figure 2 the last stable orbit, for $n=3$, is plotted versus the rotational parameter $a$. The solid enveloping curve is the result for GR. For $a=0$ the last stable orbit in GR is at $6 m$, while in pc-GR it is further in. The dark gray shaded area describes stable orbits in pc-GR and the light gray area describes unstable orbits. The pc-GR follows closely the GR with a greater deviation for larger $a$. At about $a=0.45 m$ (for $n=3$, for $n=4$ its value is a little bit larger) all orbits in pc-GR are stable up to the surface of the star, which is estimated to lie at approximately $(4 / 3) m$. For $a=m$, in GR the last stable orbit is at $r=m$.

3.2. Accretion Discs. In order to connect to actual observations [26-31], one possibility is to simulate accretion discs 


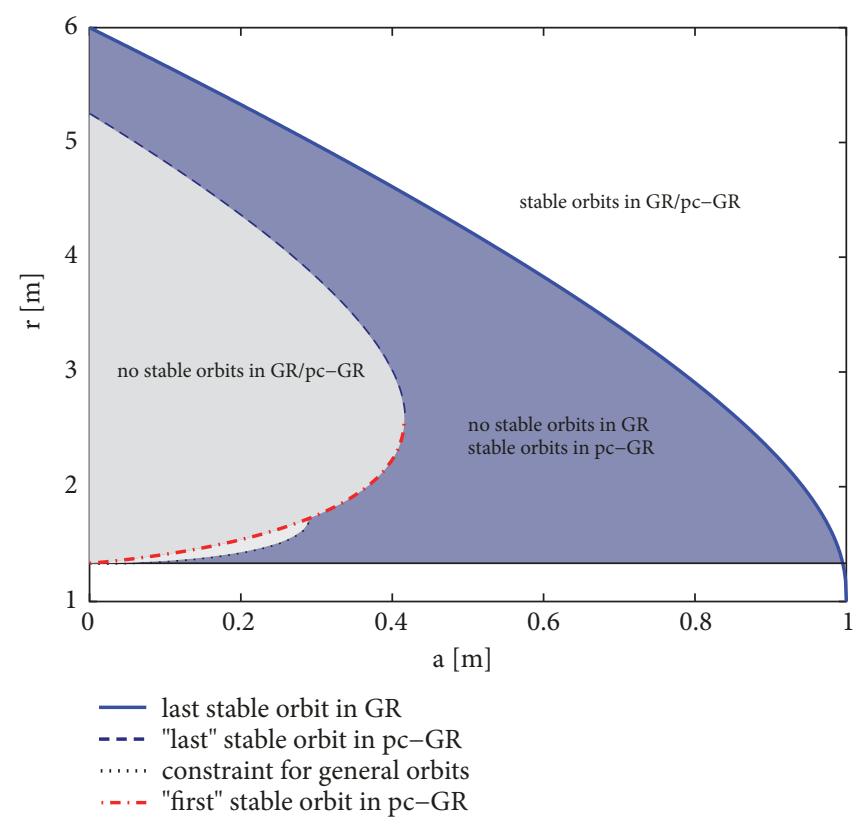

FIgURE 2: The position of the Innermost Stable Circular Orbit (ISCO) is plotted versus the rotational parameter $a$. The upper curve corresponds to GR and the lower curves correspond to pc-GR. The light gray shaded region corresponds to a forbidden area for circular orbits within pcGR. For small values of $a$ the ISCO in pc-GR follows more or less the one of GR, but at smaller values of $r$. From a certain $a$ on stable orbits they are allowed until to the surface of the star (for $n=3$ this limit is approximately $0.4 \mathrm{~m}$ and for $n=4$ it is at $0.5 \mathrm{~m}$ ).

around massive objects as the one in the center of the elliptical galaxy M87. The underlying theory was published by D. N. Page and K. S. Thorne [32] in 1974. The basic assumptions are (see also [33]) the following:

(i) A thin, infinitely extended accretion disc. This is a simplifying assumption. A real accretion disc can be a torus. Nevertheless, the structure in the emission profile will be similar, as discussed here. These discs are easier to calculate.

(ii) An energy-momentum tensor is proposed which includes all main ingredients, as mass and electromagnetic contributions.

(iii) Conservation laws (energy, angular momentum, and mass) are imposed in order to obtain the flux function, the main result of [32].

(iv) The internal energy of the disc is liberated via shears of neighboring orbitals and distributed from orbitals of higher frequency to those of lower frequency. [16].

How to deduce finally the flux is described in detail in

In order to understand within pc-GR the structure of the emission profile in the accretion disc, we have to get back to the discussion in the last subsection. The local heating of the accretion disc is determined by the gradient of orbital frequency, when going further inward (or outward). At the maximum, neighboring orbitals have nearly the same orbital frequency; thus, friction is low. On the other hand, above and especially below the position of the maximum the change in orbital frequency is large and the disc gets heated. At the maximum the heating is minimal which will be noticeable by a dark ring. Further inside, the heating increases again and a bright ring is produced.

The above consideration is relevant for $a$ larger than approximately 0.4 , as can be seen from Figure 2 (for explanations, see the figure caption) and [23]. For lower values of $a$, in pc-GR the last stable orbit follows the one of GR, but with lower values for the position of the ISCO. As a consequence, the particles reach further inside and, due to the decrease of the potential, more energy is released, producing a brighter disc. However, the last stable orbit in pc-GR does not reach $r_{\omega_{\max }}$. This changes when $a$ is a bit larger than 0.4. Now, $r_{\omega_{\max }}$ is crossed and the existence of the maximum of $\omega$ has to be taken into account as explained above.

Some simulations are presented in Figure 3. The line of sight of the observer to the accretion disc is $80^{\circ}$ (near to the edge of the accretion disc), where the angle refers to the one between the axis of rotation and the line of sight. Two rotation parameters of the Kerr solution are plotted, namely, $a=0$ (no rotation of the star, corresponding to the Schwarzschild solution) and nearly the maximal rotation $a=0.9 \mathrm{~m}$.

As a global feature, the accretion disc in pc-GR appears brighter, which is due to the fact that the disc reaches further inside where the potential is deeper, thus releasing more gravitational energy, which is then distributed within the disc.

The reason for the dark fringe and bright ring was explained above due to the variability of the friction. The dark ring is the position of the maximum of the orbital frequency. An observed position of a dark ring can, thus, be used to determine $n$. 

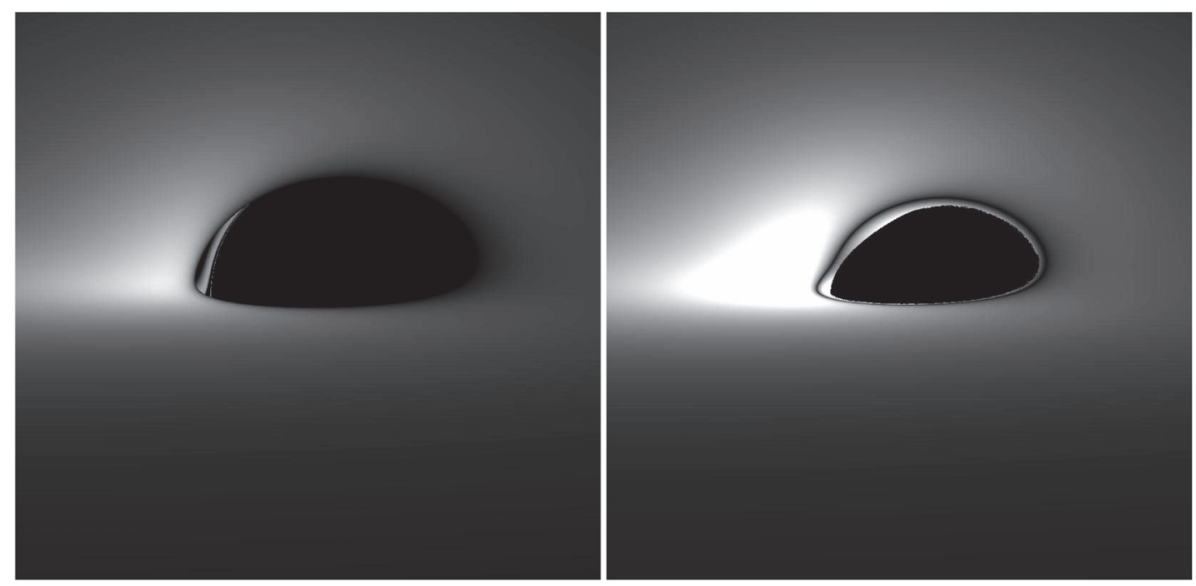

FIGURE 3: Infinite, counterclockwise rotating geometrically thin accretion disc around static and rotating compact objects viewed from an inclination of $80^{\circ}$. The left panel shows the disc model by [32] in pc-Gr, with $a=0$. The right panel shows the modified model, including pc-GR correction terms as described in the text.

The differences in the structure of an accretion disc give us clear observational criteria to distinguish between GR and pc-GR. There are still others, maybe more realistic disc models, e.g., a thick disk as described in [36]. In case there is no disk present, as is probably the case in SgrA*, then the synchrotron model of infalling and emitted gas [37] may be more realistic. However, in all of those models the above discussed ring structure of the disc will not change. Unfortunately, this is for the moment the only clear prediction to differentiate pc-GR from GR. In the next subsection we will discuss gravitational waves and we will see pc-GR and GR give different interpretations of the source, though the final outcome is the same.

Finally, in Figure 4 we compare a disc simulation for GR (left panel) with pc-GR (right panel), for $a=0.6$ and a $60^{\circ}$ inclination angle. The intensity in GR is smaller while in pcGR it is much stronger. Also, the maximum of the intensity is more in line with the EHT data, which reports the maximum at approximately 3-4m. Otherwise, the ring structure in pcGR is lost due to the low resolution of $20 \mu \mathrm{as}$ and the crossstructure of $\mathrm{pc}-\mathrm{Gr}$ is the same as in GR.

3.3. Gravitational Waves in $p c$-GR. In [4] the first observed gravitational wave event was reported. In [5] this gravitational event was investigated within the pc-GR, for $n=3$.

Using GR and the mass-point approximation for the two black holes, before the merging, a relation is obtained between the observed frequency and its temporal change to the chirping mass $\mathscr{M}_{c}$, namely [38],

$$
\mathscr{M}_{c}=\widetilde{M}_{c} F_{\omega}(r)=\frac{c^{3}}{G}\left[\frac{5}{96 \pi^{8 / 3}} \frac{d f_{\mathrm{gw}}}{d t} f_{\mathrm{gw}}^{-11 / 3}\right]^{3 / 5} .
$$

Substituting on the right hand side the observed frequency and its change and using $F_{\omega}(r)=1$ for GR, the interpretation of the source of the gravitational waves is of two black holes of about 30 solar masses each which fuse to a larger one of less than 60 solar masses. The difference in energy is radiated away as gravitational waves. However, these changes are in pc-GR, where the two black holes can come very near to each other. Unfortunately, the point mass approximation is not applicable, though in [5] this approximation was still used in order to show in which direction the interpretation of the source changes. In pc-GR $(n=3) F_{\omega}(r)=\left[1-\left(3 b_{3} / 4\right)(m / r)^{2}\right]$, which for $b_{n}$ given by the right hand side of Eq. (16) is exactly zero. Therefore, a range of the last possible distances of the two black holes before merging was assumed. On the left hand side of Eq. (20), the function $F_{\omega}(r)$ becomes very small near where the two in-spiraling black holes merge. Thus, the chirping mass $\widetilde{M}_{c}$ must be much larger than the chirping mass $\mathscr{M}$ deduced in GR. For $n=4$, the function $F_{\omega}(r)$ changes to $\left[1-\left(b_{4} / 3\right)(m / r)^{3}\right]$; thus, the main conclusions are the same, though the $r$-dependence has changed. We have not yet made explicit calculations, for one reason: The model applied in [5] has to be modified, because the point approximation is not very good.

The main result is that the source in pc-GR corresponds to two black holes with several thousand solar masses. This may be related to the merger of two primordial galaxies whose central black hole subsequently merges. One way to distinguish the two predictions is to look for light events very far way. If, for observed gravitational wave events in future, there is a consistent appearance of light events much farther away as the distance deduced from GR, then this might be in favor for pc-GR. However, all the prediction depends on the assumption that the point mass approximation is still more or less valid when the two black holes are near together, which is not very good! In [14] the inspiral frequency was determined within pc-GR, for various values of $n$, which is related to the one used in [14] by $n_{N}=n-1$. As demonstrated, the wave form cannot be reproduced satisfactorily for $n=3$; thus, it has to be increased and let us investigate the dependence of the results as a function in $n$.

In [6] the Schwarzschild case was considered and the Regge-Wheeler, for negative parity solutions, [39] and Zerrilli equations, for positive parity solutions, [40] were solved, 

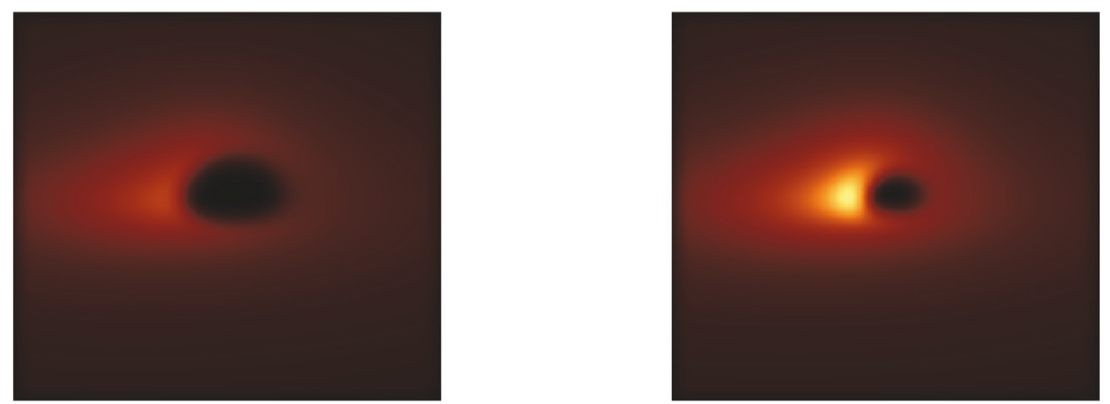

FIGURE 4: Infinite, counterclockwise rotating geometrically thin accretion disc around static and rotating compact objects viewed from an inclination of $60^{\circ}$ and $a=0.6$. The left panel is GR and the right one is pc-GR. A resolution of $20 \mu a s$ was assumed. A resolution of $20 \mu a s$ is assumed.

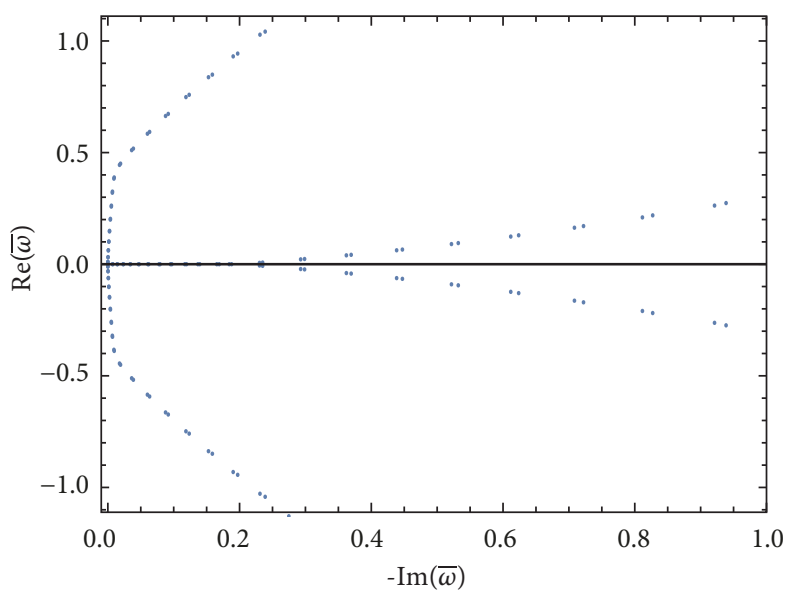

FIgURE 5: Axial gravitational modes in pc-GR. The vertical axis gives the real part of $\widetilde{\omega}=m \omega$ while the horizontal axis depicts the negative of its imaginary part.

using an iteration method [41]. Due to a symmetry, in GR the two types of solutions have the same frequency spectrum [40], which unfortunately is lost in pc-GR. For pc-GR, the spectrum of frequencies for axial modes shows a convergent behavior for the frequencies, which is shown in Figure 5. A negative imaginary part indicates a stable mode, which turns out to be the case. For an increasing imaginary part the convergence is less sure. Unfortunately, for the polar modes no convergences for the polar modes were obtained up to now.

Another problem is to distinguish between GR and pcGR. It depends very much on the observation of the ringdown frequency of the merger [6], which is not very well measured yet. Without it, we are not able to distinguish between both theories and various possible scenarios can be obtained in pc-GR $[6,12]$.

3.4. Dark Energy in the Universe. The pc-Robertson-Walker model is presented in detail in $[12,34]$. The main results will be resumed in this subsection.

The line element in gaussian coordinates has the form

$$
d \omega^{2}=(d t)^{2}-a(t)^{2} \frac{1}{\left(1+k a(t)^{2} / 4 a_{0}^{2}\right)^{2}}\left(d R^{2}\right.
$$

$$
\left.+R^{2} d \vartheta^{2}+a(t)^{2} \sin ^{2} \vartheta d \varphi^{2}\right)
$$

where $R$ is the radius of the universe and $k$ is a parameter and the energy density of matter was assumed to be homogeneous. The value $k=0$ corresponds to a flat universe, which will be taken here.

The corresponding Einstein equations were solved and an equation for the radius $a(t)$ if the universe was deduced [12]:

$$
\begin{aligned}
a(t)^{\prime \prime}= & \frac{4 \pi G}{3}(3 \beta-1) \Lambda a(t)^{3(\beta-1)+1} \\
& -\frac{4 \pi G}{3}(1+3 \alpha) \varepsilon_{0} a(t)^{-3(1+\alpha)+1}
\end{aligned}
$$

where $G$ is the gravitational constant and $\beta, \Lambda$ are parameters of the theory. The equation of state is set as $p=\alpha \varepsilon$, where $\varepsilon$ is the matter density and $\alpha$ is set to zero for dust.

Two particular solutions are shown in Figure 6. Shown is the acceleration of the universe as a function of the radius $a(t)$. The left panel shows the result for $\beta=1 / 2$ and $\Lambda=3$ and on the right hand side the parameters $\beta$ and $\Lambda$ are set to $2 / 3$ and 4 , respectively. The left figure corresponds to a solution where the acceleration tends to a constant; i.e., the 

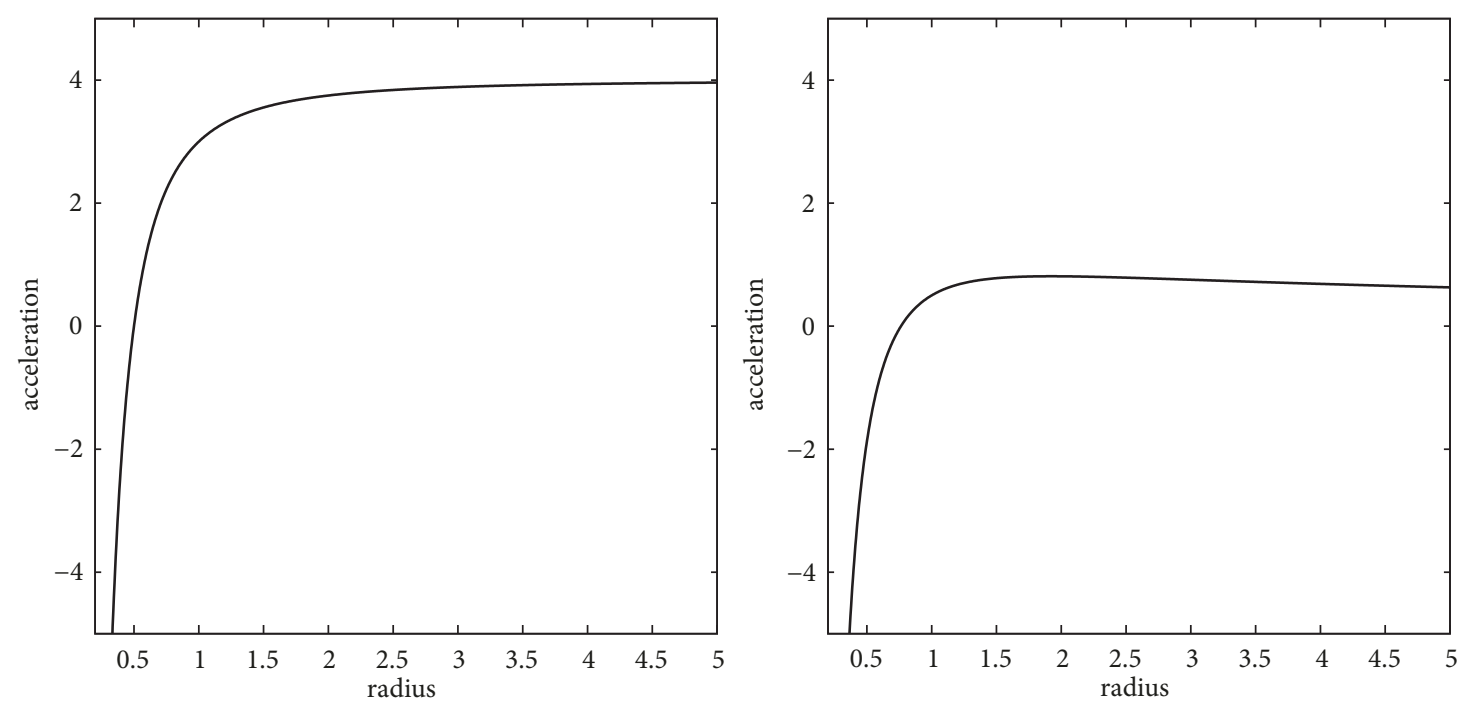

Figure 6: The left panel shows a case where the acceleration of the universe approaches a constant value and the right panel shows a case where the acceleration slowly approaches zero for $t \longrightarrow \infty$. The figures are taken from $[12,34]$.

universe will expand forever with an increasing acceleration, while in the right figure the acceleration tends slowly to zero for very large $a(t)$. In both examples the universe expands forever. These are not the only solutions; also one where the universe collapses again is possible.

These results are not very predictive, because one can obtain several possible outcomes, depending on the values of $\beta$ and $\Lambda$. Nevertheless, they show that possible scenarios for the future of our universe are still possible.

3.5. Interior of Stars. For the description of the interior of a star one needs the equation of state of matter and the coupling of the dark-energy with the matter. For the equation of state one can use the model presented in [42], which also takes into account nuclear and meson resonances. However, these approximations will lose their validity when the matter density is too large. The situation is worse for the dark-energy contribution and it is twofold: (i) one has to know how the dark-energy evolves within the star (presence of matter) and (ii) how it is coupled to the matter itself. Both are not known and we have to rest on incomplete models. Alternatively, one can approach the problem with a very interesting and distinct model to simulate the dark energy, as done in [43-46], where compact and dense objects were investigated within the pcGR and maximal masses were also deduced.

In [18] a simple coupling model of dark-energy to the mass density was proposed:

$$
\varepsilon_{\Lambda}=\alpha \rho_{m}
$$

where the index $\Lambda$ refers to the dark energy and $\rho_{m}$ refers to the mass density. In this proposal the dark energy follows neatly the mass distribution. The Tolman-OppenheimerVolkoff (TOV) equations have to be solved, which are doubled in number, one treating the mass part and the other the darkenergy part (for more details see $[12,18]$ ).
A particular result is shown in Figure 7, showing the mass of the star versus its radius. Curves are depicted for various values of the proportionality factor $\alpha$. As can be seen, the model can reproduce stable stars up to 6 solar masses, which shows that the dark-energy stabilizes stars with larger masses. However, no stars with larger masses can be constructed, because for larger values of $\alpha$ and/or larger masses the repulsion due to the dark energy becomes too large near the surface and outer surface layers are evaporated.

In [35] calculations in one-loop order, using the monopole approximation, were calculated with the intention to derive the coupling between the dark energy and matter density. In Figure 8 the lower curve shows the result of these calculations and the upper curve shows the approximation in terms of a polynomial, used in the final calculations.

Finally, in Figure 9 the mass of the star versus its radius $R$ is depicted. As can be noted, now stars with up to 200 solar masses are possible. Higher masses cannot be obtained due to the limits the model of [42] reaches.

This model also suffers from the approximations made and a complete description cannot be given. Nevertheless, now stars with up to 200 solar masses can be stabilized, which shows that the inclusion of dark-energy in massive stars may lead to stable stars of any mass! (Though, only within a phenomenological model.)

\section{Conclusions}

A report on the recent advances of the pseudocomplex General Relativity (pc-GR) was presented. The theory predicts a nonzero energy-momentum tensor on the right hand side of the Einstein equation. The new contribution is related to vacuum fluctuations, but due to a missing quantized theory of gravitation one recurs to a phenomenological ansatz. Calculations in one-loop order, with a constant back-ground 


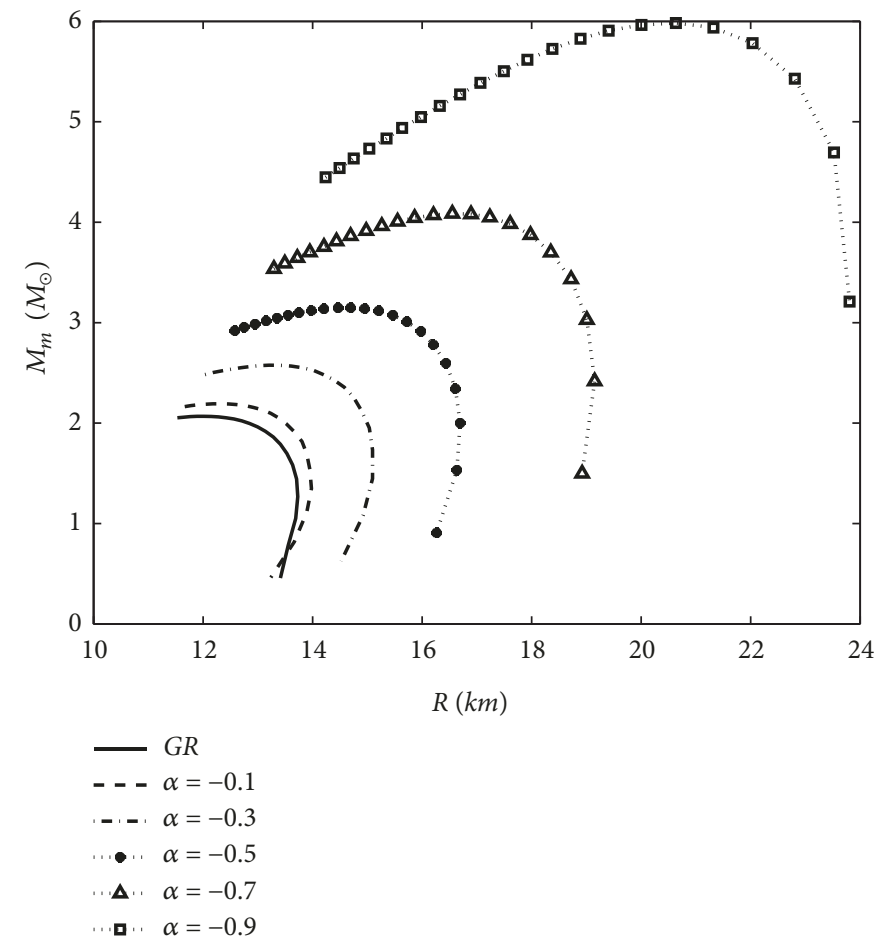

FIgURE 7: The figure shows the dependence of the mass of the star as a function of its radius (figure taken from [18]).

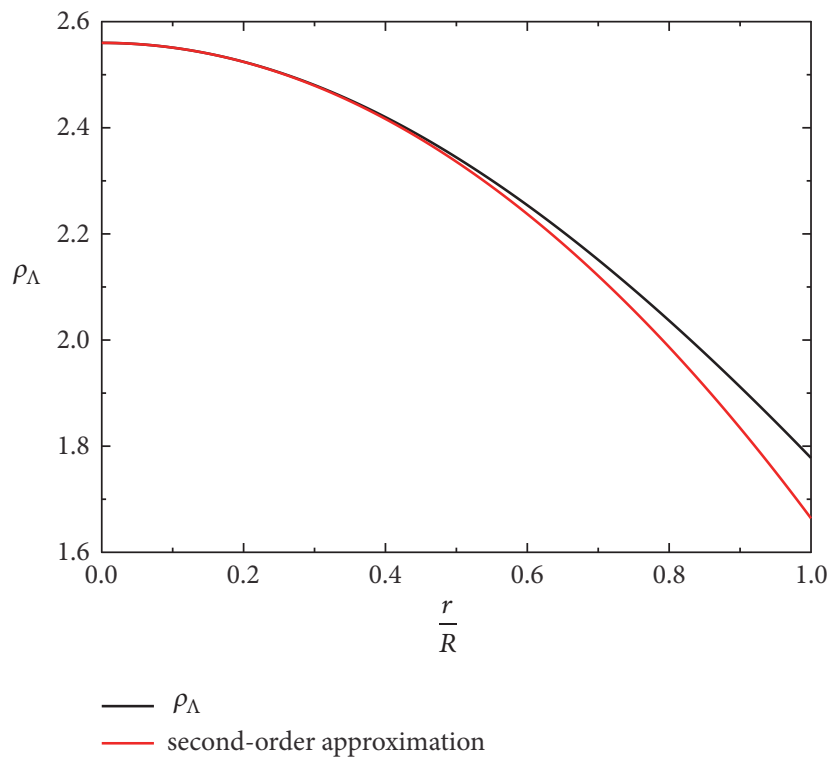

FIGURE 8: Dark energy density as a function of $r / R$, where $R$ is the radius of the star. The upper curve depicts the result of the monopole approximation and the lower curve is an approximation for the upper one. The figure is taken from [35].

metric, show that the dark energy density has to increase toward smaller $r$.

Consequences of the theory were presented: (i) the appearance of a dark ring followed by a bright one in accretion discs around black holes, (ii) a new interpretation of the source of the first gravitational event observed, (iii) possible outcomes of the future evolving universe, and (iv) attempts to stabilize stars with large masses.
The only robust prediction is the structure in the emission profile of an accretion disc.

\section{Conflicts of Interest}

The author declares that they have no conflicts of interest. 


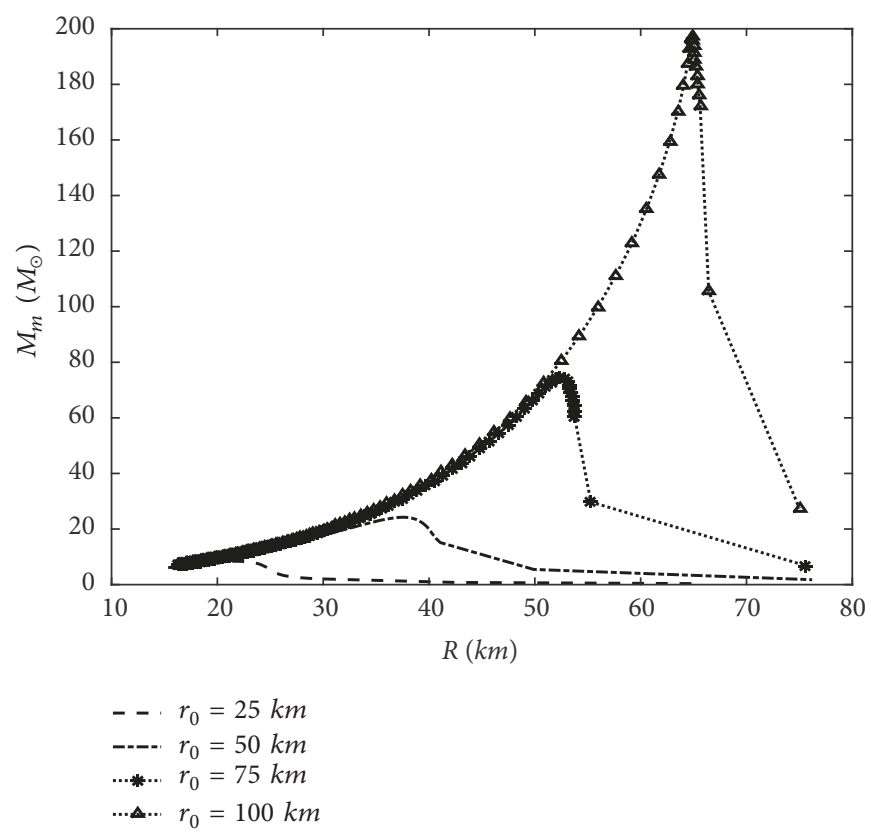

Figure 9: The mass of a star as a function on its radius $R$. With the modified coupling of the dark-energy to the mass density the maximal mass possible is now about 200. The figure is taken from [35].

\section{Acknowledgments}

Peter O. Hess acknowledges the financial support from DGAPA-PAPIIT (IN100418). Very helpful discussions with T. Boller (Max-Planck Institute for Extraterrestrial Physics, Garching, Germany) and T. Schönenbach are also acknowledged.

\section{References}

[1] C. W. Misner, K. S. Thorne, and J. A. Wheeler, Gravitation, W. H. Freeman and Company, San Francisco, Calif, USA, 1973.

[2] C. M. Will, "The confrontation between general relativity and experiment," Living Reviews in Relativity, vol. 9, no. 3, 2006.

[3] J. M. Weisberg, J. H. Taylor, and L. A. Fowler, "Gravitational waves from an orbiting pulsar," Scientific American, vol. 245, no. 4, p. 74, 1981.

[4] B. P. Abbott, R. Abbott, T. D. Abbott et al., "Observation of gravitational waves from a binary black hole merger," in Physical Review Letters, vol. 116, LIGO Scientific Collaboration and Virgo Collaboration, 2016.

[5] P. O. Hess, "The black hole merger event GW150914 within a modified theory of general relativity," Monthly Notices of the Royal Astronomical Society, vol. 462, no. 3, pp. 3026-3030, 2016.

[6] P. O. Hess, "Regge-wheeler and zerilli equations within a modified theory of general relativity," Astron. Nachr, vol. 340, no. 1-3, pp. 89-94, 2019.

[7] A. Einstein, "A generalization of the relativistic theory of gravitation," Annals of Mathematics: Second Series, vol. 46, pp. 578-584, 1945.

[8] A. Einstein, "A generalized theory of gravitation," Reviews of Modern Physics, vol. 20, pp. 35-39, 1948.

[9] M. Born, "A suggestion for unifying Quantum Theory and Relativity," Proceedings of the Royal Society London. Series A:
Mathematical and Physical Sciences, vol. 165, no. 921, pp. 291303, 1938.

[10] M. Born, "Reciprocity Theory of Elementary Particles," Reviews of Modern Physics, vol. 21, no. 3, pp. 463-473, 1949.

[11] E. R. Caianiello, "Is there a maximal acceleration?" Lettere al Nuovo Cimento, vol. 32, no. 3, pp. 65-70, 1981.

[12] P. O. Hess, M. Schäfer, and W. Greiner, Psuedo-Complex General Relativity, Springer, Heidelberg, Germany, 2016.

[13] P. F. Kelly and R. B. Mann, "Ghost properties of algebraically extended theories of gravitation," Classical and Quantum Gravity, vol. 3, no. 4, pp. 705-712, 1986.

[14] A. Nielsen and O. Birnholz, "Testing pseudo-complex general relativity with gravitational waves," Astronomical Notes, vol. 339, p. 298, 2018.

[15] P. O. Hess and W. Greiner, "Pseudo-complex general relativity," International Journal of Modern Physics E, vol. 18, p. 51, 2009.

[16] P. O. Hess and W. Greiner, Centennial of General Relativity: A Celebration, C. A. Z. Vasconcellos, Ed., World Scientific, Singapore, 2017.

[17] R. Adler, M. Bazin, and M. Schiffer, Introduction to General Relativity, McGraw-Hill, New York, NY, USA, 2nd edition, 1975.

[18] I. Rodríguez, P. O. Hess, S. Schramm, and W. Greiner, "Neutron stars within pseudo-complex General Relativity," Journal of Physics G, vol. 41, Article ID 105201, 2014.

[19] N. D. Birrell and P. C. W. Davies, Quantum Fields in Curved Space, Cambridge University Press, Cambridge, UK, 1986.

[20] M. Visser, "Gravitational vacuum polarization. III. Energy conditions in the (1+1)-dimensional Schwarzschild spacetime," Physical Review D: Particles, Fields, Gravitation and Cosmology, vol. 54, no. 8, pp. 5116-5122, 1996.

[21] G. Caspar, T. Schönenbach, P. O. Hess, M. Schäfer, and W. Greiner, "Pseudo-complex General Relativity: Schwarzschild, Reissner-Nordström and Kerr solutions," International Journal of Modern Physics E, vol. 21, Article ID 1250015, 2012. 
[22] T. Schönenbach, $\mathrm{PhD}$ thesis, Universität Frankfurt am Main, Germany, 2014.

[23] T. Schönenbach, G. Caspar, P. O. Hess et al., "Experimental tests of pseudo-complex general relativity," Monthly Notices of the Royal Astronomical Society, vol. 430, no. 4, pp. 2999-3009, 2013.

[24] T. Boller, P. O. Hess, A. Müller, and H. Stöcker, "Predictions of the pseudo-complex theory of gravity for EHT observations - I. Observational tests," Monthly Notices of the Royal Astronomical Society, vol. 485, no. L34, 2019.

[25] P. O. Hess, T. Boller, A. Müller, and H. Stöcker, "Predictions of the pseudo-complex theory of Gravity for EHT observations - II: theory and predictions," Monthly Notices of the Royal Astronomical Society, vol. 482, no. L121, 2019.

[26] The Event Horizon Telescope collaboration, "First M87 event horizon telescope results. v. physical origin of the asymmetric ring," The Astrophysical Journal Letters, vol. 875, no. L1, 2019a.

[27] The Event Horizon Telescope collaboration, "First M87 event horizon telescope results. v. physical origin of the asymmetric ring," The Astrophysical Journal Letters, vol. 875, no. L2, $2019 \mathrm{~b}$.

[28] The Event Horizon Telescope collaboration, "First M87 event horizon telescope results. v. physical origin of the asymmetric ring," The Astrophysical Journal Letters, vol. L3, 2019c.

[29] The Event Horizon Telescope collaboration, "First M87 event horizon telescope results. v. physical origin of the asymmetric ring," The Astrophysical Journal Letters, vol. 14, 2019d.

[30] The Event Horizon Telescope collaboration, "First M87 event horizon telescope results. v. physical origin of the asymmetric ring," The Astrophysical Journal Letters, vol. 875, no. L5, 2019e.

[31] The Event Horizon Telescope collaboration, "First M87 event horizon telescope results. v. physical origin of the asymmetric ring," The Astrophysical Journal Letters, vol. 875, no. L6, $2019 \mathrm{f}$.

[32] D. N. Page and K. S. Thorne, "Disk-accretion onto a black hole. time-averaged structure of accretion disk," Astrophysical Journal, vol. 191, pp. 499-506, 1974.

[33] T. Schönenbach, G. Caspar, P. O. Hess et al., "Ray-tracing in pseudo-complex General Relativity," Monthly Notices of the Royal Astronomical Society, vol. 442, no. 1, pp. 121-130, 2014.

[34] P. O. Hess, L. Maghlaoui, and W. Greiner, "The RobertsonWalker metric in a pseudo-complex General Relativity," International Journal of Modern Physics E, vol. 19, p. 1315, 2010.

[35] G. Caspar, I. Rodríguez, P. O. Hess, and W. Greiner, "Vacuum fluctuation inside a star and their consequences for neutron stars, a simple model," International Journal of Modern Physics E, vol. 25, Article ID 1650027, 2016.

[36] W. Kluzniak and S. Rappaport, "Magnetically torqued thin accretion disks," The Astrophysical Journal, vol. 671, no. 2, p. 1990, 2007.

[37] K. Dodds-Eden, D. Porquet, G. Trap et al., "Evidence For X-ray synchrotron emission from simultaneous mid-infrared to Xray observations of a strong Sgr A* flare," Astronomical Physical Journal, vol. 698, p. 676, 2009.

[38] M. Maggiore, GravitationalWaves, vol. 1, Oxford Univ. Press, Oxford, UK, 2008.

[39] T. Regge and J. A. Wheeler, "Stability of a Schwarzschild singularity," Physical Review A: Atomic, Molecular and Optical Physics, vol. 108, no. 4, pp. 1063-1069, 1957.

[40] S. Chandrasekhar, The Mathematical Theory of Black Holes, The Clarendon Press, New York, NY, USA, 1st edition, 1983.

[41] H. Cho, A. S. Cornell, J. Doukas, T.-R. Huang, and W. Naylor, "A new approach to black hole quasinormal modes: a review of the asymptotic iteration method," Advances in Mathematical Physics, vol. 2012, Article ID 281705, 2012.

[42] V. Dexheimer and S. Schramm, "Proto-neutron and neutron stars in a Chiral SU(3) model," The Astrophysical Journal, vol. 683, p. 943, 2008.

[43] G. L. Volkmer, Um Objeto Compacto Excotico na Relatividade Geral Pseudo-Complexa [Ph. D. thesis], Porto Alegre, Brazil, March 2018.

[44] M. Razeira, D. Hadjimichef, M. V. T. Machado, F. Köpp, G. L. Volkmer, and C. A. Z. Vasconcellos, "Effective field theory for neutron stars with WIMPS in the pc-GR formalism," Astronomische Nachrichten, vol. 338, p. 1073, 2017.

[45] D. Hadjimichef, G. L. Volkmer, R. O. Gomes, and C. A. Z. Vasconcellos, Memorial Volume: Walter Greiner, P. O. Hess and H. Stöcker, Eds., World Scientific, Singapore, 2018.

[46] G. L. Volkmer and D. Hadjimichef, "Mimetic dark matter in pseudo-complex General Relativity," International Journal of Modern Physics: Conference Series, vol. 45, Article ID 1760012, 2017. 

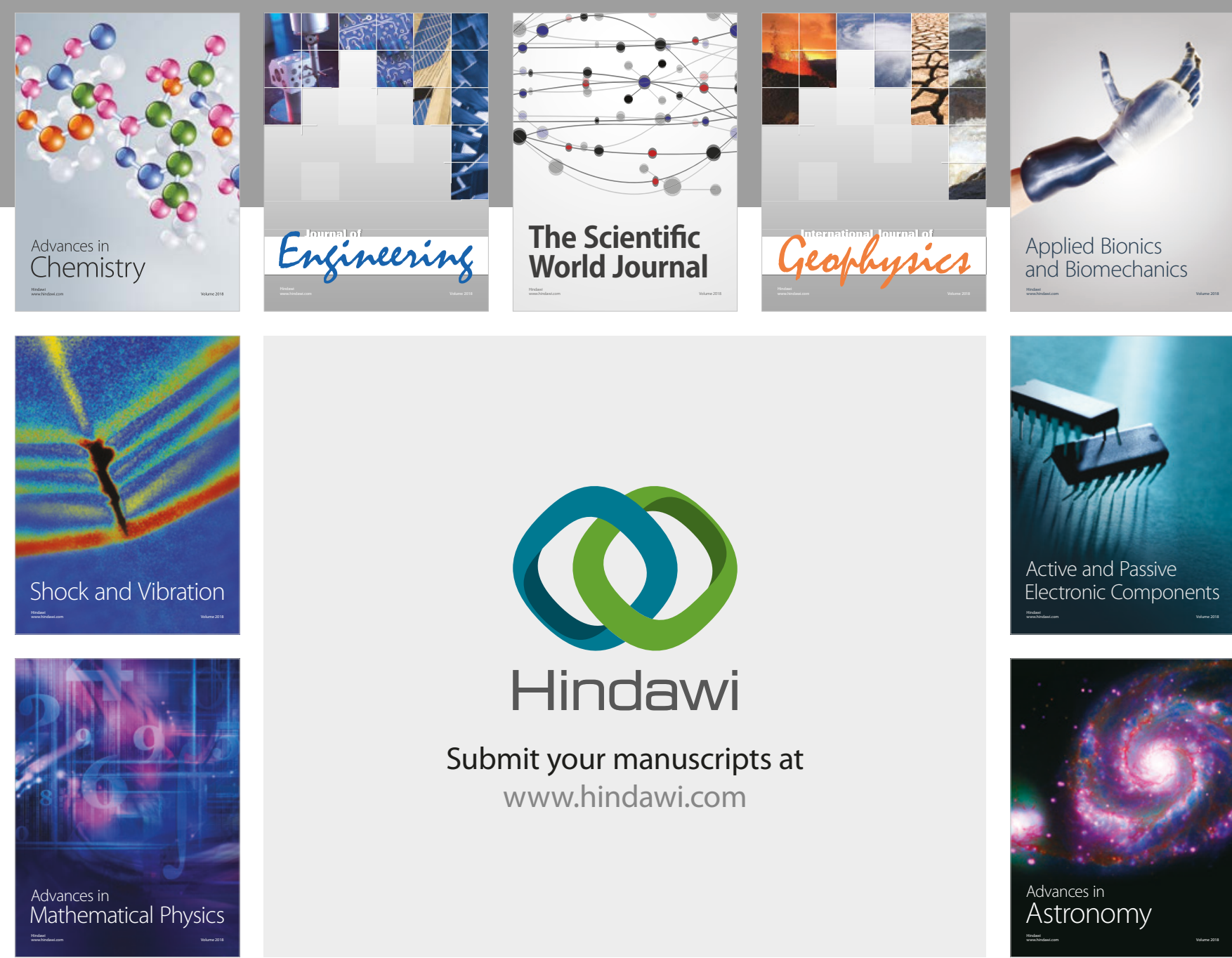

Submit your manuscripts at

www.hindawi.com

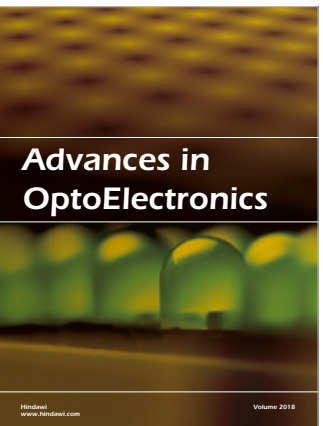

\section{Rotcting Machinery}
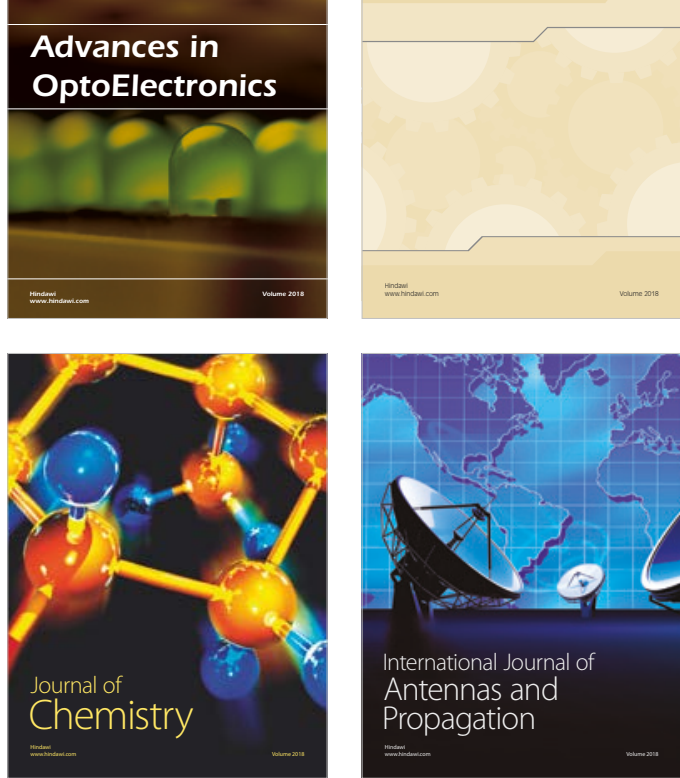

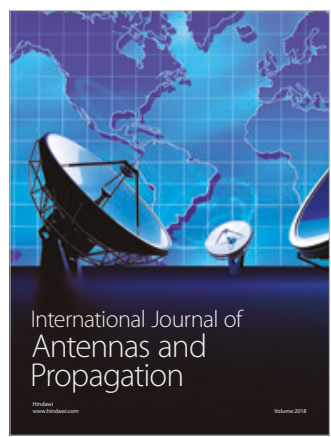

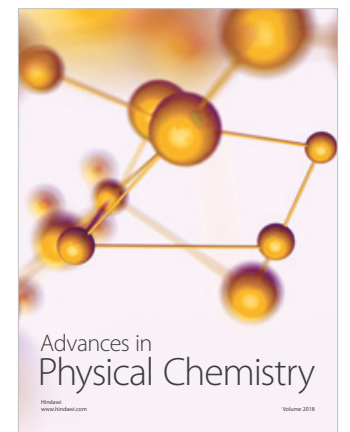

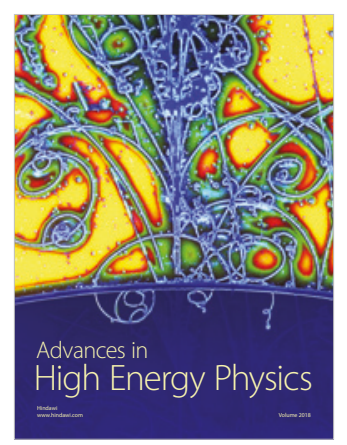

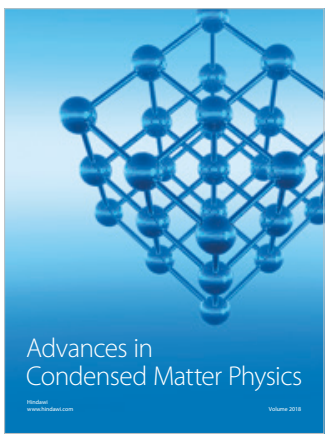

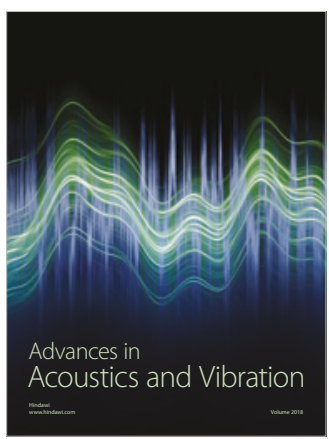

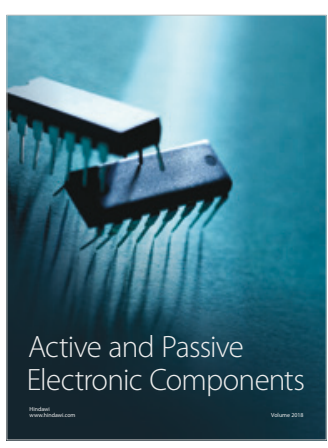
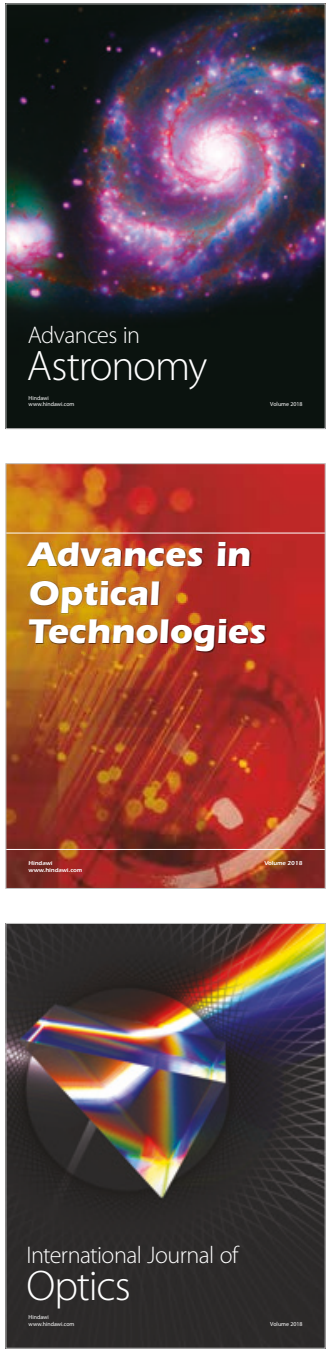